\title{
Response of central nervous system aspergillosis to voriconazole
}

\author{
Prakash Balasubramaniam, Pranesh B. Madakira, Anish Ninan, Aarthi Swaminathan \\ Department of Neurology, PSG Institute of Medical Sciences and Research, Avinasi Road, Peelamedu, Coimbatore - 641002 , India
}

Fungal infections of the central nervous system (CNS) usually present as subacute meningitis. Other manifestations include mass effect and focal neurological deficits. ${ }^{[1]}$ Cerebrospinal fluid (CSF) examination and biopsy of the lesion are helpful in disclosing the organism involved. Aspergillosis presents as brain abscess or granuloma with predominant neutrophils in CSF. Voriconazole is a broad spectrum triazole antifungal agent. It can be given orally and has lesser adverse effects We report a 69-years-old diabetic male, with aspergilloma of para-nasal sinus invading the CNS, who responded well to voriconazole treatment. He discontinued the medication by himself as it was costly. Within a month of stopping the medication, he developed features of subacute meningitis. However he showed clinical improvement after the medication was restarted. The case is reported for the clinical evidence of antifungal activity of voriconazole against aspergillosis.

Key words: Aspergilloma, aspergillosis, fungal granuloma, voriconazole

\section{Introduction}

Most of the fungi are non pathogenic for healthy humans. Occasionally they reach the bloodstream, meninges or brain. In the CNS, the fungi either produce meningitis, meningo-encephalitis or focal infection (abscess or granuloma). Zygomycetes and Aspergillus may invade blood vessel leading to thrombosis. ${ }^{[1]}$ Central nervous system fungal infections are less reported and the incidence varies by geographic location and immunological state. Seventy five percent of patients with CNS fungal infections have serious underlying medical conditions affecting the immune system. Males, AIDS patients, Blacks, Hispanics and Asians are infected more than others. ${ }^{[1]}$

Voriconazole is a broad spectrum triazole antifungal which has potent activity against aspergillosis.

\section{Case Report}

A 69-years-old male, who was a poorly controlled diabetic for twenty years, developed copious nasal secretions which contained black particles. A few weeks later he started having left frontal and maxillary ache. Examination revealed left eye refractory error (6/9) and afferent pupillary defect suggestive of optic nerve lesion. Extra ocular movements (EOM) and fundii were normal. Nose was congested with mucosal mass. No other neurological deficits could be made out. Blood investigations revealed hemoglobin-12.1 gm\%, total white blood cell counts-9000 cells $/ \mathrm{mm}^{3}$ (N-62\%, L-35\%, E-3\%), blood sugar-198 mg\%, urea-20 mg\%, creatinine-1.0 mg\% and $\mathrm{HbA}_{1 \mathrm{C}}-9.4 \%$. A plain and contrast CT scan and MRI [Figure 1] of the brain and sinuses showed irregular contrast enhancing soft tissue lesion of the left maxillary sinus extending into the orbit and middle cranial fossa $(19 \times 18 \mathrm{~mm})$. The histopathology of the tissue from the maxillary sinus showed fungal elements with thick mycelium and large septate hyphae. Left ethmoidectomy was done, fungal elements were cleared and the pus was released.

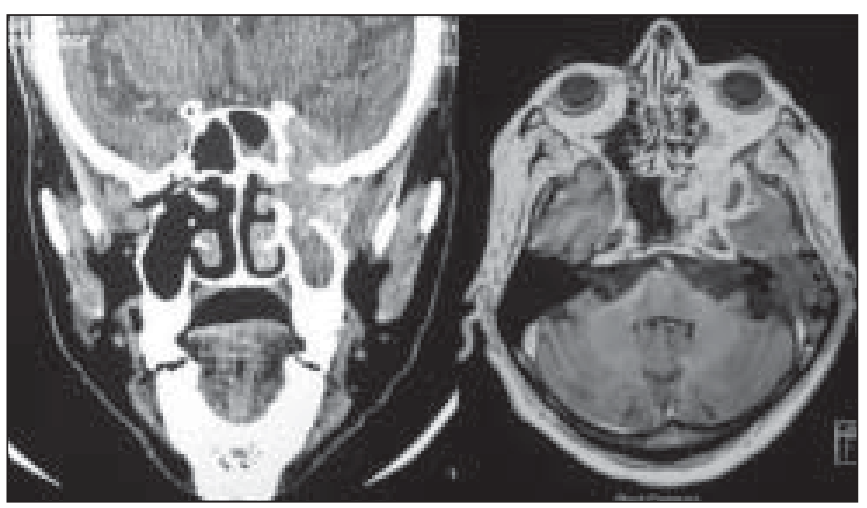

Figure 1: CT scan (Plain, Coronal) and MRI (Axial, Gadolinium contrast) showing the enhancing soft tissue mass extending from the maxillary sinus into the middle cranial fossa and to orbital apex. 
The duramater was found open and was repaired. The histopathological examination (HPE) showed giant cells with branching hyphae consistent with Aspergillus fumigatus and the same was grown in culture. After the surgery he deteriorated, developed excruciating left hemicranial headache. A repeat CT scan showed residual sphenoidal sinusitis. He had been prescribed itraconazole $100 \mathrm{mg}$ bid per-oral. Twenty days after this he presented to us.

His complaints were severe left hemicranial ache and high fever. His left eyelid gradually drooped over a period of 15 days. There was no history of limb weakness or tremors. Examination showed a toxic apathetic individual, who was mildly confused; however he could answer simple questions. There was total ptosis of the left eye. On opening, there was conjunctival injection, absent vision and the pupil being $4 \mathrm{~mm}$ not reacting to light. Extra ocular movements were absent in all directions. Examination of the jaw, face, palate, tongue and motor system were normal. All modalities of sensations were retained. No in-coordination was noted. Cardiovascular, respiratory and per abdomen examinations were normal.

Investigations showed leucocytosis, hyperglycemia $(248 \mathrm{mg} / \mathrm{dl})$ and raised erythrocyte sedimentation rate (115 mm/h). Repeat CT scan [Figure 2] done 45 days after the first CT, showed enhancing soft tissue mass in left the sphenoid and maxillary sinus orbital apex, para-pharyngeal space and, lateral wall of nasopharynx. Bony defects were noted in the left greater wing of the sphenoid orbital apex and maxillary sinus. Left basal ganglia and temporal lobe had showed edema. The size of the intra cranial mass was $25 \times 20 \mathrm{~mm}$.

Itraconazole $100 \mathrm{mg}$ per-oral bid and intravenous liposomal amphotericin-B $50 \mathrm{mg}$ on alternate days were given for 10 days. He required haloperidol for restlessness. As there was no improvement, itraconazole was changed over to voriconazole $200 \mathrm{mg}$ per oral bid. His sensorium and EOM gradually improved over a period of three weeks. He could be discharged healthy except for impaired vision and minimal extra ocular

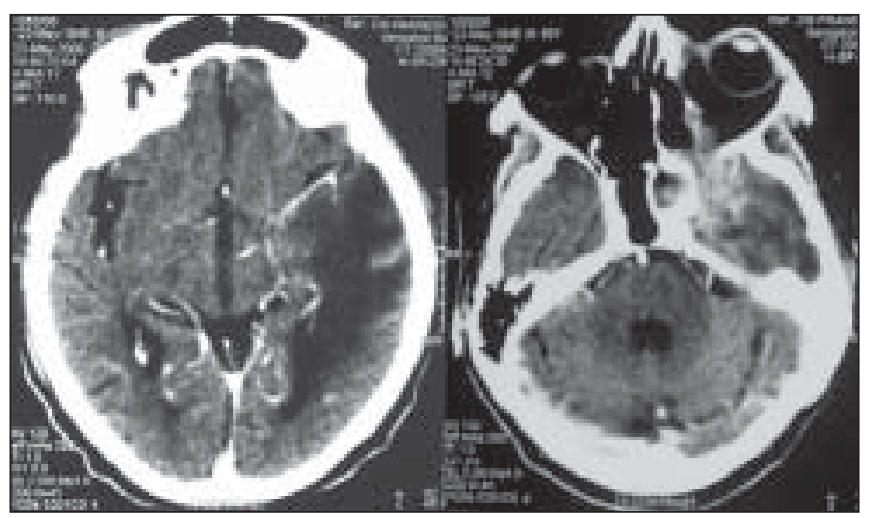

Figure 2: CT scan brain contrast 60 days after surgery showing worsening of lesion with gross cerebral edema. paresis of the left eye. There was significant reduction in the size of mass as evidenced by CT scan (10x13 mm). At the time of discharge he was on voriconazole $200 \mathrm{mg}$ per oral bid oral hypoglycemic agents and carbamazepine $300 \mathrm{mg} /$ day and other supportive measures. He gradually improved and started leading a normal life. The ptosis, proptosis and EOM became normal. The pain was reduced and nasal discharge cleared. He had mild left maxillary sinus tenderness. The metabolic parameters were normal. As the voriconazole was costly he discontinued it after 75 days. He presented one month later with mild fever, confusion, language disturbance and was swaying to the right side. His left pupil was $4 \mathrm{~mm}$ and the right was $2 \mathrm{~mm}$ and he developed right facial weakness. A CT scan showed [Figure 3] contrast enhancing mass $(22 \times 30 \mathrm{~mm})$ with a fresh cystic component (10x11 mm). He was admitted and found to be restless, aphasic and incontinent. His vitals were normal and voriconazole $200 \mathrm{mg}$ per oral bid was restarted. He improved gradually over the next three months and is well for the next six months except for loss of vision in the left eye. He is being followed up.

\section{Discussion}

Central nervous system Aspergillosis was first reported in 1897, is increasing in prevalence and is common in India due to environmental factors. The commonest risk factor was diabetes, the commonest route was from a contiguous site and the commonest pathology was granuloma. ${ }^{[2]}$ If it spreads haematogenously into the brain, it causes diffuse inflammation, cerebral edema, micro-abscesses and septic hemorrhagic infarcts. There is a tendency of fibrosis and granuloma formation if it spreads by local invasion. It also invades the blood vessels and occludes it. The ENT or ophthalmological symptoms last for several months, before the creeping cerebral infection. The CT and MRI scans of brain are the most helpful in localizing lesions. The usual drug of choice is amphotericin B. Voriconazole is a newer triazole with broad spectrum antifungal activity. It acts against various fungi including Aspergillus species. ${ }^{[3]}$ It is

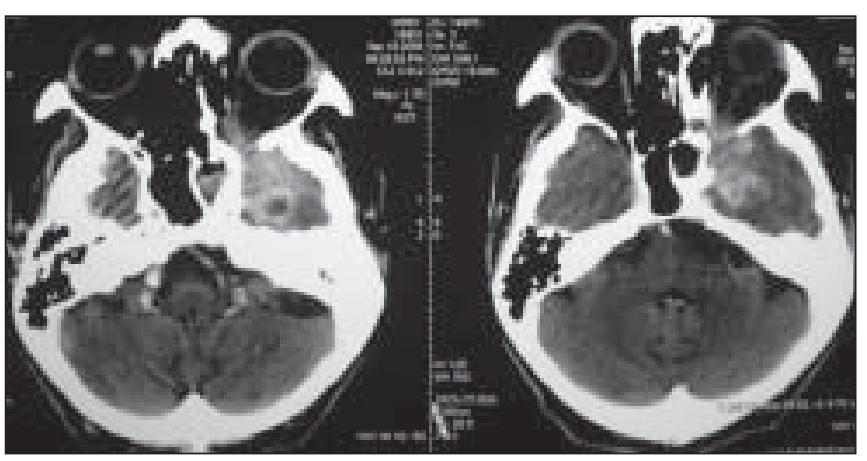

Figure 3: Contrast CT brain 30 days after stopping voriconazole showing cystic component 
available both as oral and intravenous (IV) formulations. Its bioavailability is $95 \%$, distributed into most of the body fluids including CSF. It has fewer adverse effects that include photopsia, rash and hepatitis. The IV formulation should not be used in patients with renal failure with creatinine clearance of less than $50 \mathrm{ml} / \mathrm{min}$ to prevent accumulation of the IV vehicle sulpho hydroxyl propyl beta cyclodextrin. Its efficacy against aspergillosis is better than amphotericin in terms of response rate, survival rate, safety and tolerability. ${ }^{[4]}$ A superior antifungal activity was noted by combining voriconazole with amphotericin than with monotherapy. ${ }^{[5]}$ Voriconazole is a suitable alternative or combination therapy to amphotericin B for the treatment of invasive CNS aspergillosis. Other extended spectrum triazoles like posaconazole which has a good activity against Aspergillus species, may be reserved for refractory cases. ${ }^{[6]}$ Echinocandins like caspofungin have good activity against aspergillosis but their CSF penetration is poor.

Here we have presented an elderly diabetic patient with CNS aspergillosis, who had a good response to voriconazole.

\section{References}

1. Davis LE. Fungal infections of the central nervous system. Neurol Clin 1999;17:761-81.

2. Sundaram C, Umabala P, Laxmi V, Purohit AK, Prasad VS, Panigrahi M, et al. Pathology of fungal infections of the central nervous system: 17 years experience from Southern India. Histopathology 2006;49:396-405.

3. Schwartz S, Thiel E. CNS-Aspergillosis: Are there new treatment options? Mycoses 2003;46:8-14.

4. Herbrecht R, Denning DW, Patterson TF, Bennett JE, Greene RE, Oestmann JW, et al. Voriconazole versus amphotericin B for primary therapy of invasive aspergillosis. N Engl J Med 2002;347:408-15.

5. Clemons KV, Espiritu M, Parmar R, Stevens DA. Comparative efficacies of conventional amphotericin b, liposomal amphotericin B (AmBisome), caspofungin, micafungin, and voriconazole alone and in combination against experimental murine central nervous system aspergillosis. Antimicrobial Agents Chemother 2005;49:4867-75.

6. Walsh TJ, Raad I, Patterson TF, Chandrasekar P, Donowitz GR, Graybill $\mathrm{R}$, et al. Treatment of invasive aspergillosis with posaconazole in patients who are refractory to or intolerant of conventional therapy: An externally controlled trial. Clin Infect Dis 2007;44:2-12.

Accepted on 14-04-2007

Source of Support: Nil, Conflict of Interest: None declared. 